\title{
Fluid Flow Characteristics in Micro-Pump with the Aid of Peltier Devices and Thermal Expansion Material
}

\author{
Shuichi Torii and Yasuhiro Takakura \\ Graduate School of Science and Technology, Kumamoto University, Kumamoto 860-8555, Japan
}

Received: December 28, 2015 / Accepted: January 12, 2016 / Published: February 29, 2016.

\begin{abstract}
Experimental study is performed to design and develop a cylindrical micro-pump driven by expansion and contraction of the heat deformation material, whose variation is caused with the aid of heating and cooling properties of Peltier devices. The pump consists of the diffuser valve unit, the heat deformation material unit, the nozzle valve unit, the Peltier devices and the cover. The input current of the Peltier devices is controlled by the bipolar power supply so that the Peltier devices are heated and cooled periodically. The working fluid flow in the micro-pump is caused by the periodical thermal deformation of material which is caused by the periodical heating and cooling of the Peltier devices. In order to measure the fluid flow in the micro-pump, micro air bubbles are employed as a tracer. The corresponding movement is recorded by X-ray apparatus and its velocity is measured by PIV (particle image velocimetry). It is found that, the micro-pump developed here can make the working fluid flow. The corresponding fluid flow in the micro pump is confirmed by the numerical method.
\end{abstract}

Key words: Flow visualization, micropump, Peltier device, X-ray.

\section{Introduction}

The local injection medical treatment and the intraarterial injection chemotherapy have attracted attention as a dosage method of the anticancer agent. The local injection medical treatment is a method to administer a medicine like anticancer drug directly in a tumor with a needle, and to be able to do without patients' strong side effects. The intraarterial injection chemotherapy is a method to administer an anticancer drug directly in an artery. Its advanced characteristic is to attenuate the side effect, because only a little amount of anticancer drug is used by circulation of the artery through a body.

The wafer type gas chromatography which had invented in Stanford University in 1970's, is known as the first MEMS (micro electro mechanical systems) application apparatus [1], and is the origin of the micro fluid device. The micro pump is one of the application research and development fields of the

Corresponding author: Shuichi Torii, professor, research field: thermal fluid engineering.
MEMS technology [2]. Its fundamental device and mechanics are proposed from the end of the 1980's to the beginning of the 1990's with the MEMS technology development. In addition, the flow control of the micro fluid is substantially modified with rapid development of TAS (micro total analysis system) which is a fusion domain of analytic chemistry and MEMS technology. The diaphragm type pump is the earliest micropump with the use of MEMS technology which is developed by van Lintel [3] in 1988. However, there is a problem of structure because of the complexity of the manufacturing process due to a movable check valve. The pump which is a simple structure having a diffuser valve is reported by Stemme [4]. This pump is used as an operation principle of the micropump. Tsai [5] studies the valve less pump operated by a thermal bubble.

The concept of the present study is to insert both micropump and microtank near the cancer tumor in the human body. Here, the micropump is operated by a thermoelectric element from a temperature 
difference between the body temperature and the temperature outside of body, resulting in an injection of an anticancer agent from micropump. As a first step, the present study is to construct the cylinder prototype pump operated with the aid of thermal deformation materials and Peltier devices. Consideration is given to the operating condition of the micropump and the flow characteristics in the pump which are disclosed by the flow visualization method and X-ray apparatus. The corresponding flow characteristics are confirmed by the numerical simulation.

\section{Pumping Principle}

Fig. 1 depicts a schematic of the micropump which consists of the nozzle valve unit, the diffuser valve unit, the thermal deformation material unit, Peltier devices and stainless cover. Here, the cross-section of the pump is a cylinder in shape. Both sides of the main body, i.e., the thermal deformation material part are connected with the nozzle valve and the diffuser valve, respectively. The regular expansion and compression motions of the thermal deformation material are periodically driven with the aid of the
Peltier device, resulting in the flow of the working fluid. Here the Peltier device is controlled by bipolar power supply (BP4610, NF Circuit Design Block Corporation, Japan). In other words, when the outer surface of the thermal deformation material is heated by the Peltier device, its material causes thermal expansion, resulting in an attenuation of internal diameter of the micro pump. In contrast, when the thermal deformation material is cooled, the internal diameter increases. These both opposite motions are periodically repeated with the aid of the bipolar power supply, as mentioned in the following. By the periodical motion of the heat deformation material, the working fluid inside the pump flows due to a loss difference between the shapes of the diffuser valve and the nozzle valve.

Table 1 summarizes the linear expansion rate of various materials. High-polymer materials and polyoxymethylene resin (POM and polyacetal resin) are recommended as a thermal deformation material because of higher linear expansion rate. Here the polyoxymethylene resin is employed as the material of the micropump and the corresponding thermal properties are summarized in Table 2.

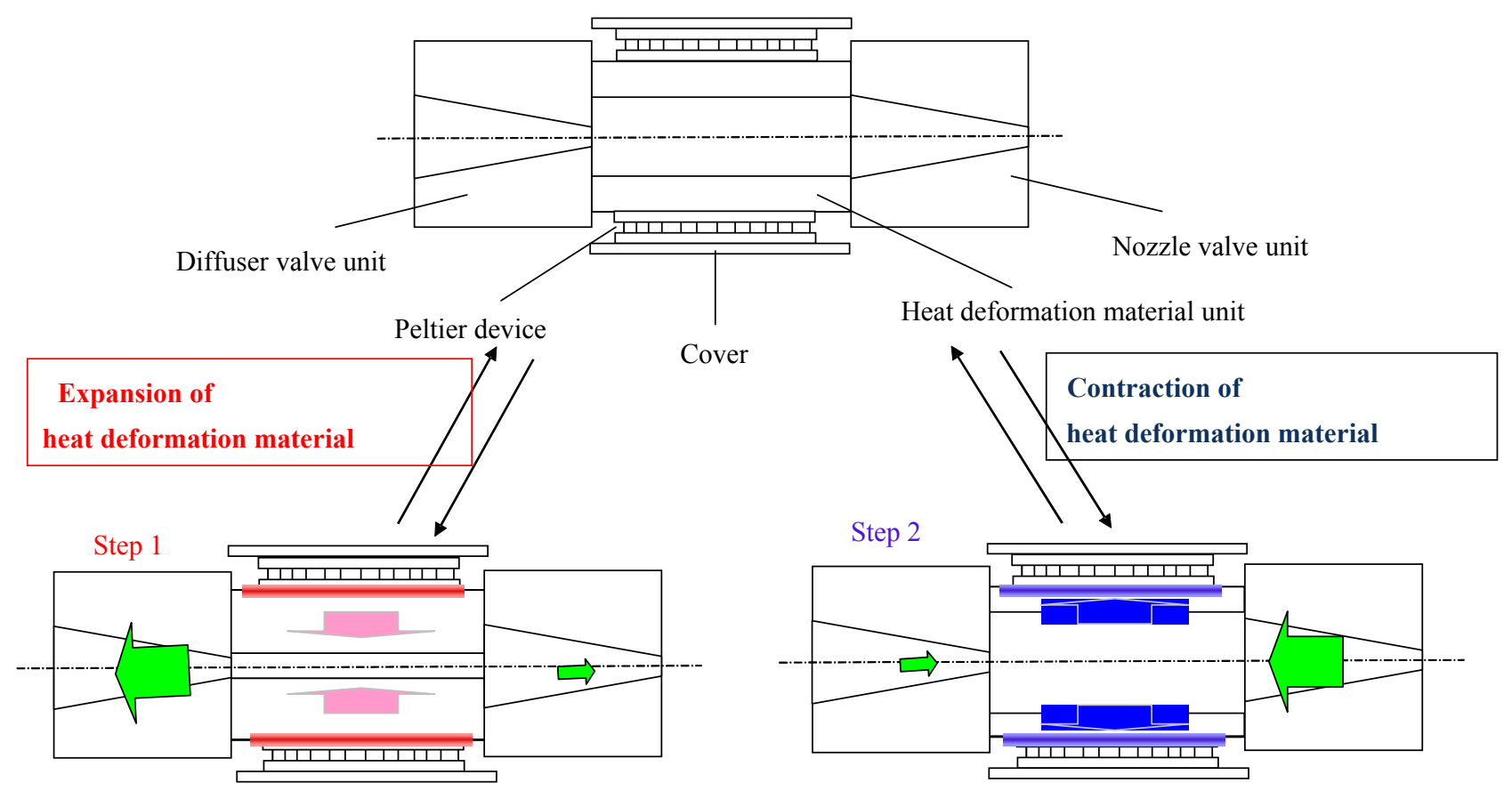

Fig. 1 Cross-section of micro-pump and its operation. 
Table 1 Difference between some materials' coefficients of liner expansion.

\begin{tabular}{ll}
\hline Material & Coefficient of liner expansion $\left(\times 10^{-6} /{ }^{\circ} \mathrm{C}\right)$ \\
\hline Iron & 11.8 \\
Copper & 16.7 \\
Aluminium & 23.0 \\
Elastic rubber & 77 \\
Polyethylene & $55-100$ \\
Polyester & $100-200$ \\
\hline
\end{tabular}

Table 2 Properties of polyoxymethylene.

\begin{tabular}{ll}
\hline Material & Polyoxymethylene \\
\hline Melting point $\left({ }^{\circ} \mathrm{C}\right)$ & 165 \\
Specific heat $\left(\mathrm{J} /\left(\mathrm{kg} \cdot{ }^{\circ} \mathrm{C}\right)\right)$ & 1,470 \\
Coefficient of liner expansion $\left(\times 10^{-6} /{ }^{\circ} \mathrm{C}\right)$ & $90-130$ \\
Thermal conductivity $\left(\mathrm{W} /\left(\mathrm{m} \cdot{ }^{\circ} \mathrm{C}\right)\right)$ & 0.23 \\
\hline
\end{tabular}

\section{Experimental Apparatue and Method}

Fig. 2 illustrates the experimental apparatus which consists of a micropump, a Peltier device, X-ray devices and bipolar power supply. The thermal deformation material unit used here is periodically heated or cooled by the Peltier device which is controlled by the bipolar power supply. As mentioned previously, this material deformation causes the fluid flow in the micropump. In order to observe this flow, the X-ray apparatus (MX-90Basic made, MediXtec Company, Japan) is used. The specification is summarized in Table 3. PIV (particle image velocimetry) measurement is employed to confirm a responsibility of the micropump developed here and the fluid flow characteristics through the pump. The pump which includes the particle-liquid mixture, is installed on the X-ray apparatus table. $\mathrm{X}$-rays are irradiated and at the same time, the internal structure deformation of the thermal deformation material part and the movement of particles in the pump is photographed and recorded in PC. By using the digital data in PC, the PIV analysis is performed to obtain the particle velocity and motion with time. In the pre-experiment, aluminum powder, glass beads, and loam balloons, magnesium pieces and air bubbles were used as a tracer. In the present study, water and air bubbles are employed as hydraulic fluid and tracer, respectively.

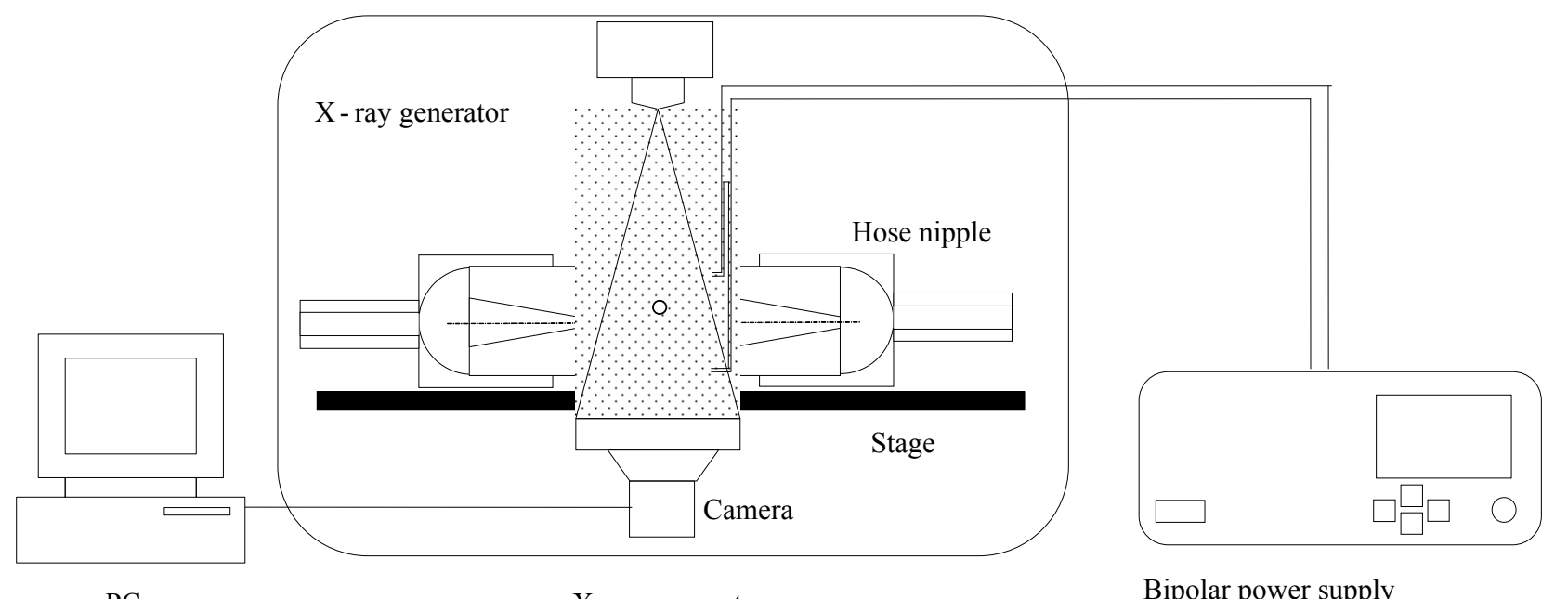

PC

$\mathrm{X}$ - ray apparatus

Bipolar power supply

Fig. 2 Experimental apparatus. 
Table 3 Specifications of X-ray apparatus.

\begin{tabular}{ll}
\hline Model & MX-90 basic \\
\hline Voltage of X-ray tube & Max $90 \mathrm{kV}$ \\
X-ray tube current & Max $0.5 \mathrm{~mA}$ \\
Focal spot size of X-ray tube & $0.1 \mathrm{~mm}$ \\
X-ray detector & X-ray charge coupled device camera \\
Dimension of input plane & $24 \times 18 \mathrm{~mm}$ \\
Resolution & 13 line pea $/ \mathrm{mm}$ \\
X-ray leakage dose & Under $10 \mathrm{~Sv} / \mathrm{h}$ \\
\hline
\end{tabular}

Table 4 Experimental condition.

\begin{tabular}{lll}
\hline & & Experimental condition \\
\hline Input current $(\mathrm{A})$ & & $\pm 0.6(0.06 \mathrm{~V})$ \\
Input frequency $(\mathrm{Hz})$ & $1 / 60$ \\
\multirow{2}{*}{ X-ray apparatus } & Voltage of X-ray tube $(\mathrm{kV})$ & 47.5 \\
& X-ray tube current $(\mathrm{mA})$ & 0.508 \\
\hline
\end{tabular}

An experimental condition in the pump response experiment is shown in Table 4. An uncertainty analysis [6] yields the following results: the uncertainty for the water flow rate is estimated to be $\pm 2.5 \%$ and that of the time interval measurement is $\pm 1.0 \%$. Since the uncertainty in the physical properties of less than $\pm 1 \%$ plays only a minor effect.

\section{Governing Equations and Numerical Method}

The proposed physical model to be studied and the coordinate system are schematically illustrated in Fig. 3. The following assumptions are imposed in the formulation of the problem: incompressible, steady state, uniform inlet velocity and uniform inlet fluid temperature. Under these assumptions, the governing equations for mass, momentum and energy are described in the tensor formation as:

Continuity equation:

$$
\frac{\partial U_{i}}{\partial x_{i}}=0
$$

Momentum equation:

$$
U_{j} \frac{\partial U_{i}}{\partial x_{j}}=-\frac{1}{\rho} \frac{\partial P}{\partial x_{i}}+v \frac{\partial}{\partial x_{j}}\left[\left(\frac{\partial U_{i}}{\partial x_{j}}+\frac{\partial U_{j}}{\partial x_{i}}\right)\right]
$$

where, $U_{i}$ is velocity component in the $x_{i}$ direction, $\rho$ is density, and $v$ is molecular viscosity. The flow can be assumed to obey the two dimensional Navier-Stokes equations for laminar incompressible flow in the axisymmetric coordinate system. The governing equations employed here are discretized using the finite difference methods. The numerical cord employed here is developed in our laboratory. The system variables $P$, and $U_{i}$ are calculated with a staggered grid. A computation reveals a small difference, i.e., maximum $2 \%$, when the grid system is changed from $400 \times 800$ to $800 \times 1,600$. Hence, a grid system of $400 \times 800$ with uniformly distributed nodal points is employed in the present study to save computation time. The nonslip boundary condition is employed on the surface of the diamond cylinders. Numerical computation was performed at time interval $\Delta t=0.0001 \mathrm{~s}$ using water as the working fluid. Simulations with various different grids, as mentioned previously, were conducted to determine the grid-independent solutions. Throughout the calculation range considered here, the maximum relative error was estimated to be about $2 \%$ by comparing the solutions on regular and fine grids with twice of the grid points. Although a few solutions were computed with half the time step to ensure consistency and time-step independence, there was no substantial discrepancy between two different time intervals. 


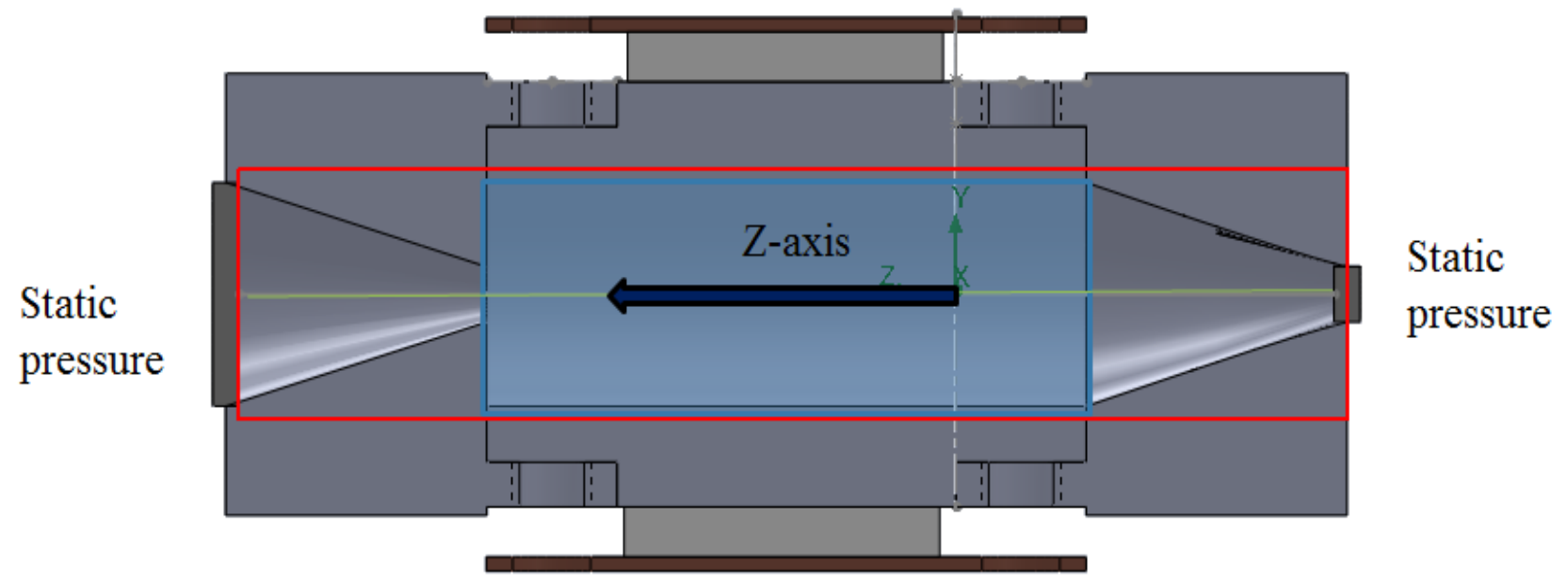

Fig. 3 Coordinate system.

\section{Results and Discussion}

Fig. 4 depicts an example of the response of the control side of the Peltier device. The bipolar power supply gives the pulsating input voltage, resulting in the oscillate deformation of the material. By using its regular oscillating deformation of the material, air bubbles as tracer particles is gradually moved with the distilled water as shown in Fig. 5. Here, the white color is bubble. In general, for one cycle of pump's

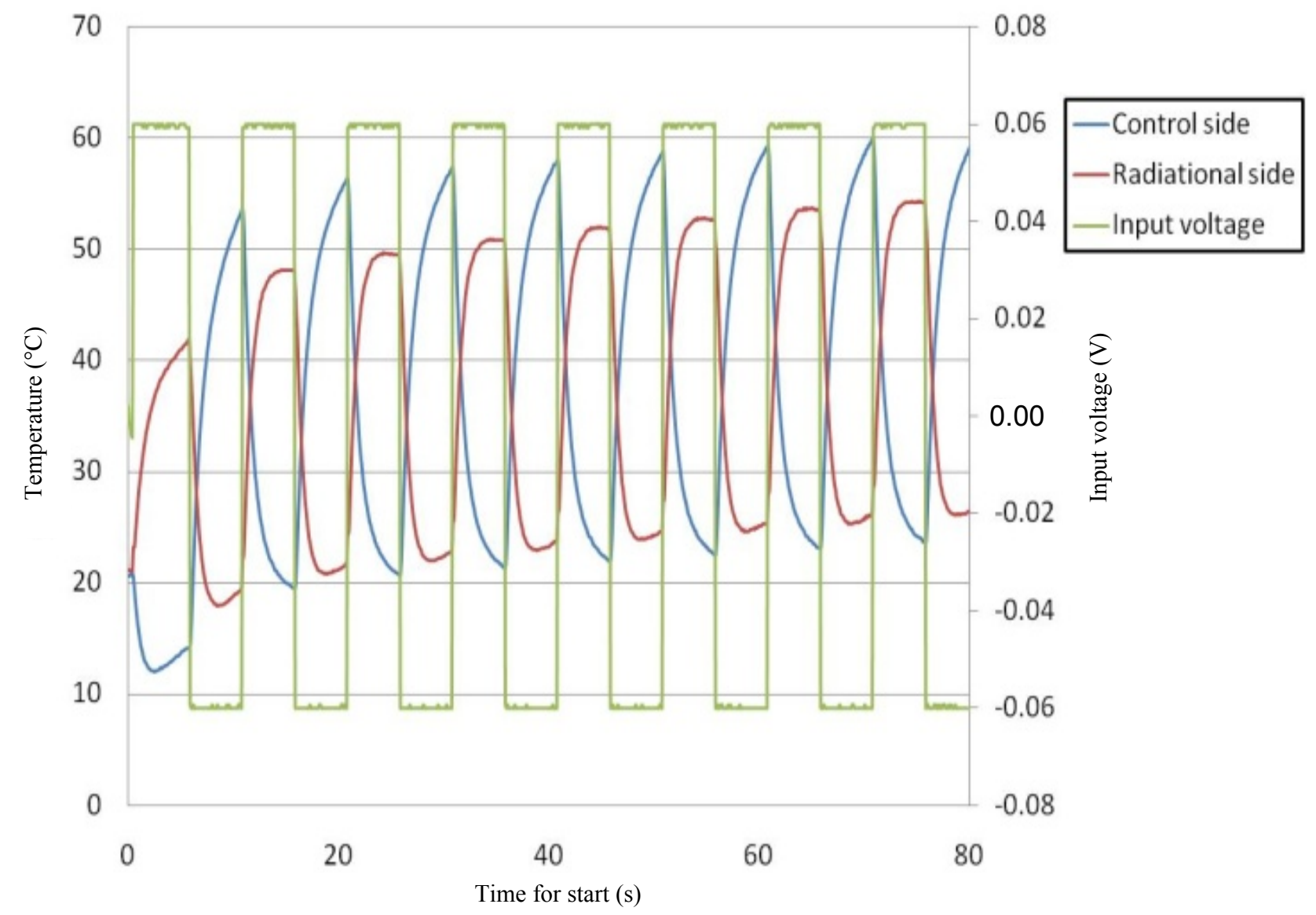

Fig. 4 Responsibility of Peltier device. 


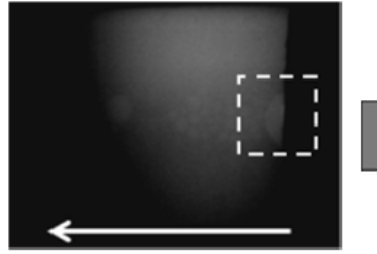

Flow direction

Fig. 5 Moving of air bubbles inside the pump.

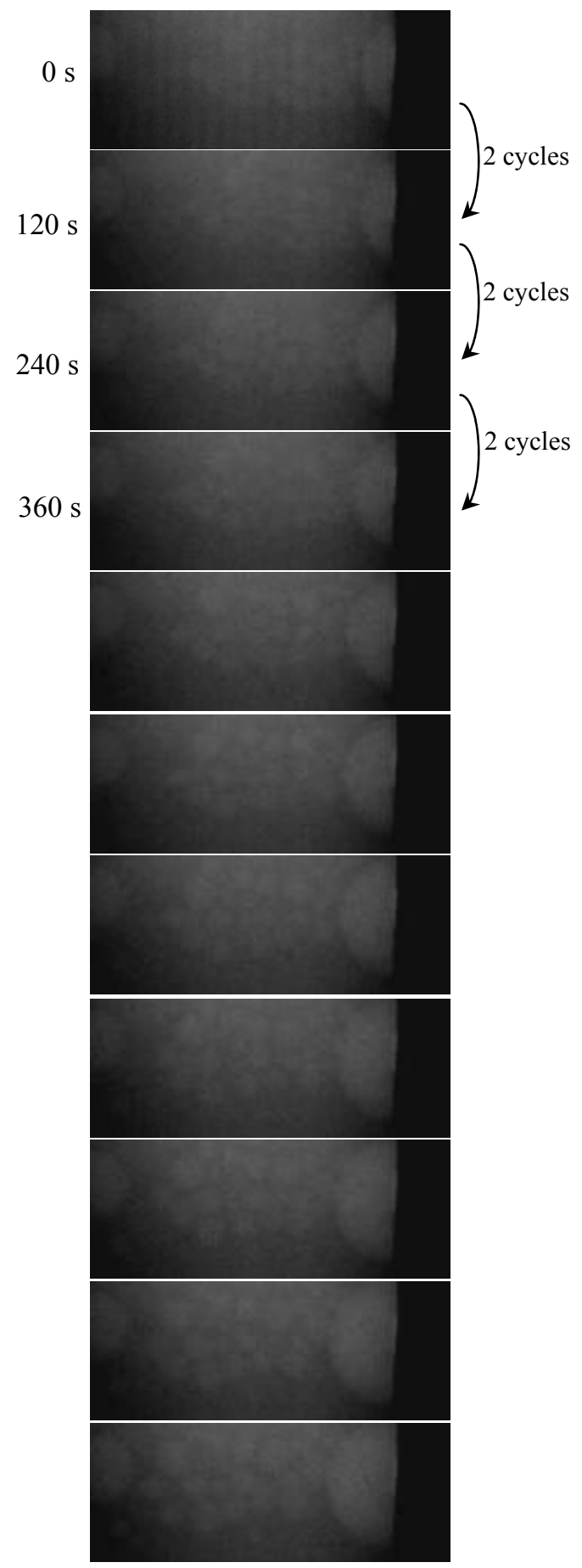

Fig. 6 Moving of air bubbles per 2 cycles.

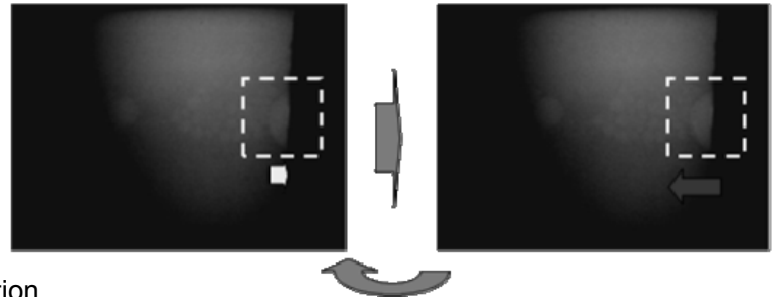

moving, air bubble goes backward and goes forward and consequently, the regular movement causes gradually the forward motion of a bubble. Fig. 6 depicts that, air bubble moves one direction gradually, i.e., in the direction of right side to left side. The speed estimated based on the timewise motion of an air bubble was about $1.55 \mathrm{~mm} / \mathrm{s}$ which corresponds to the fluid velocity.

Fig. 7 illustrates the flow velocity distribution in the cross-section of the micro-pump and the velocity profile along Z-axis when the deformation of the material is heated. One observes that, the positive flow velocity yields at the diffuser valve due to contraction of the inner wall of the micro-pump, while the negative velocity appears at the nozzle valve. Based on the velocity profile, the mass flow rate is estimated. The corresponding numerical result is depicted in Fig. 8 when the deformation of the material is cooled. The fluid flow is in contrast to that in Fig. 7. In other words, although the fluid flows along the Z-axis at the nozzle valve, the fluid in the vicinity of the diffuser valve is sucked into the micro-pump. Note that, the maxim velocity in Figs. 7 and 8 becomes larger than that in the experiment, as mentioned above. This is because since the micro-pump size in the experiment becomes smaller than that in the numerical simulation, the relatively high velocity is predicted than the experimental result.

Both velocity profiles in Figs. 7 and 8 are superimposed in Fig. 9. It is found that, both profiles periodically take place, resulting in a fluid movement along Z-axis. The fluid flow concept in micro-pump, as mentioned previously, is qualitatively predicted by the numerical method. 


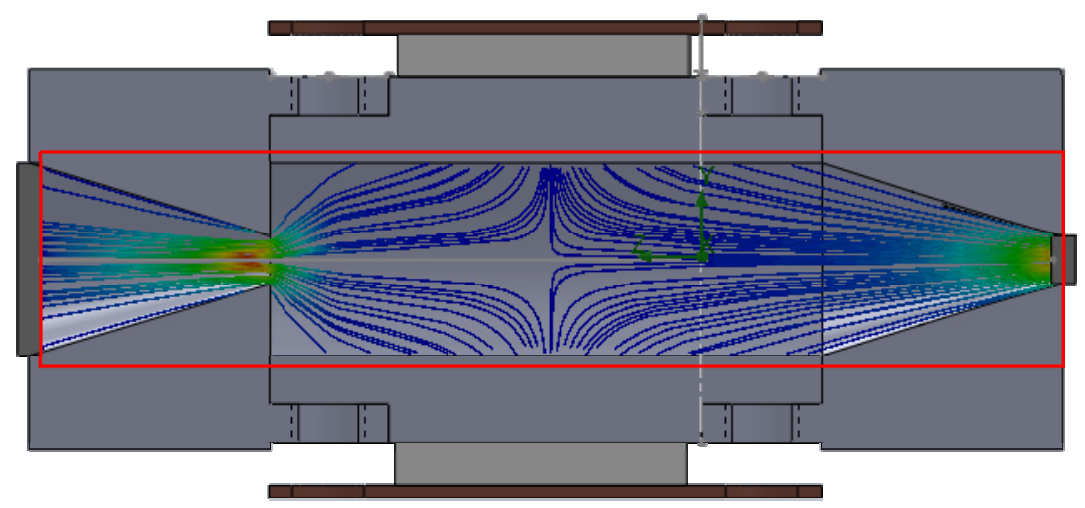

(a) Velocity distribution

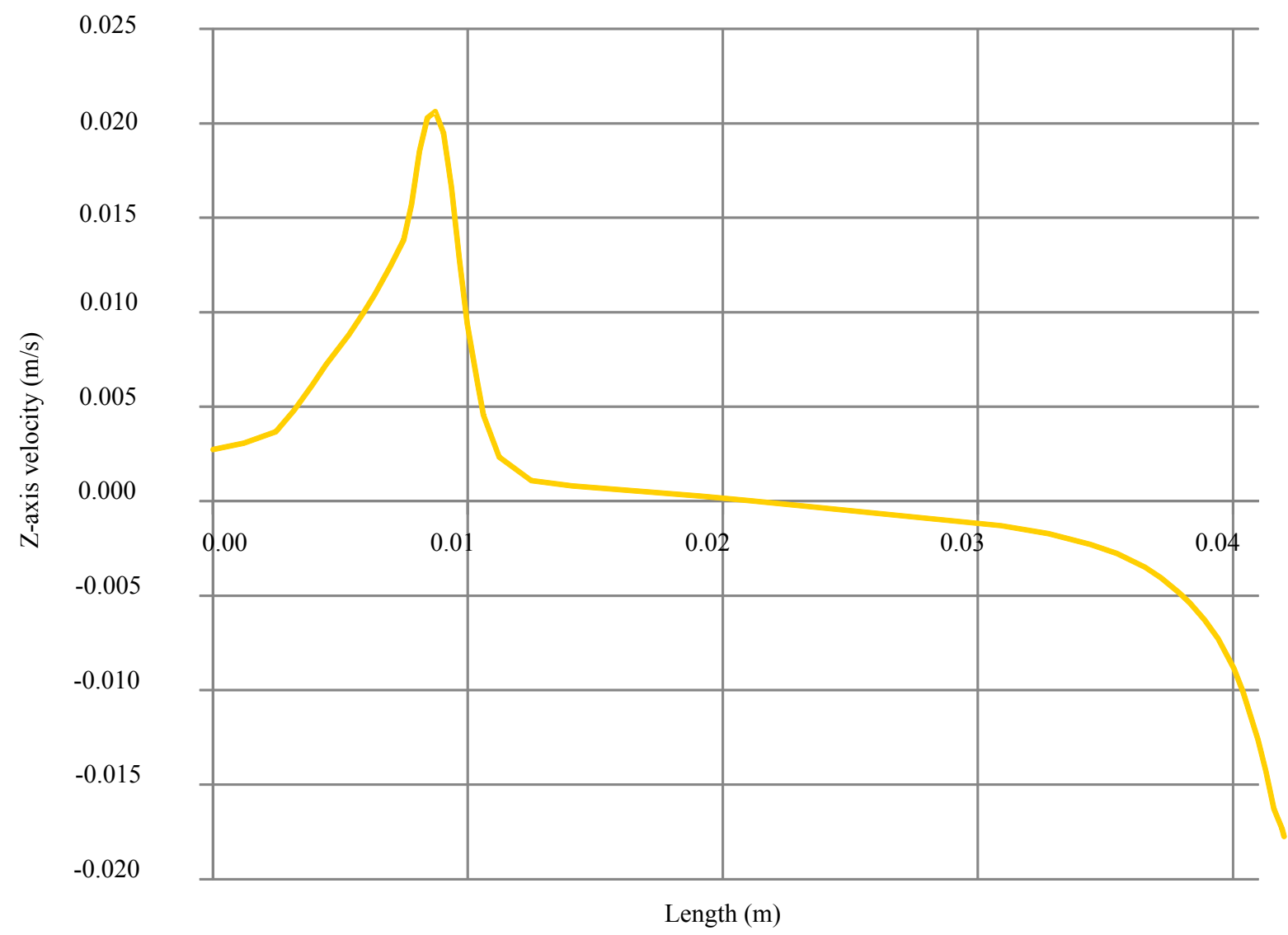

(b) Velocity profile at Z-axis

Fig. 7 Numerical results in the micro-pump in which the heat deformation of material is heated. 


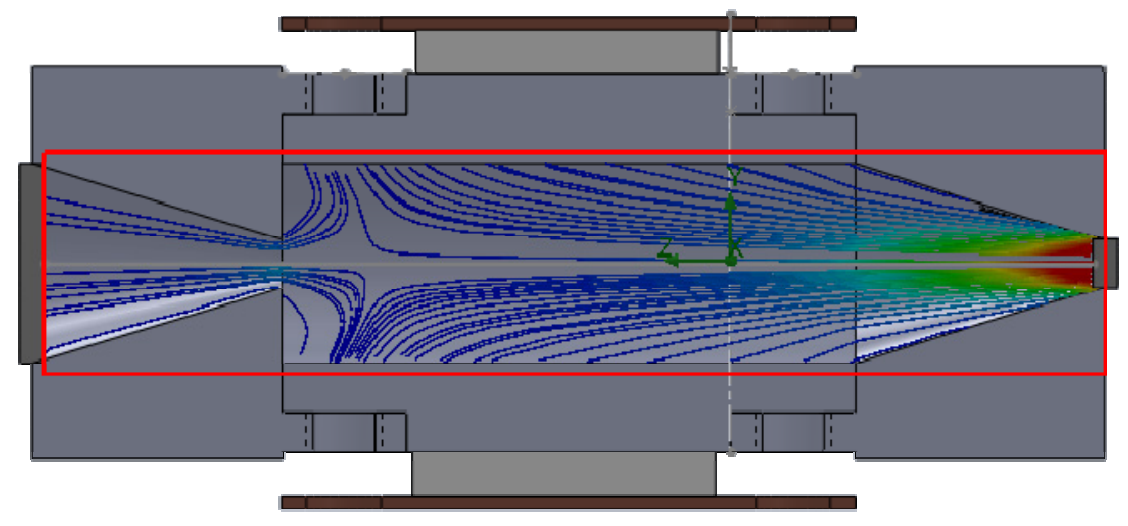

(a) Velocity distribution

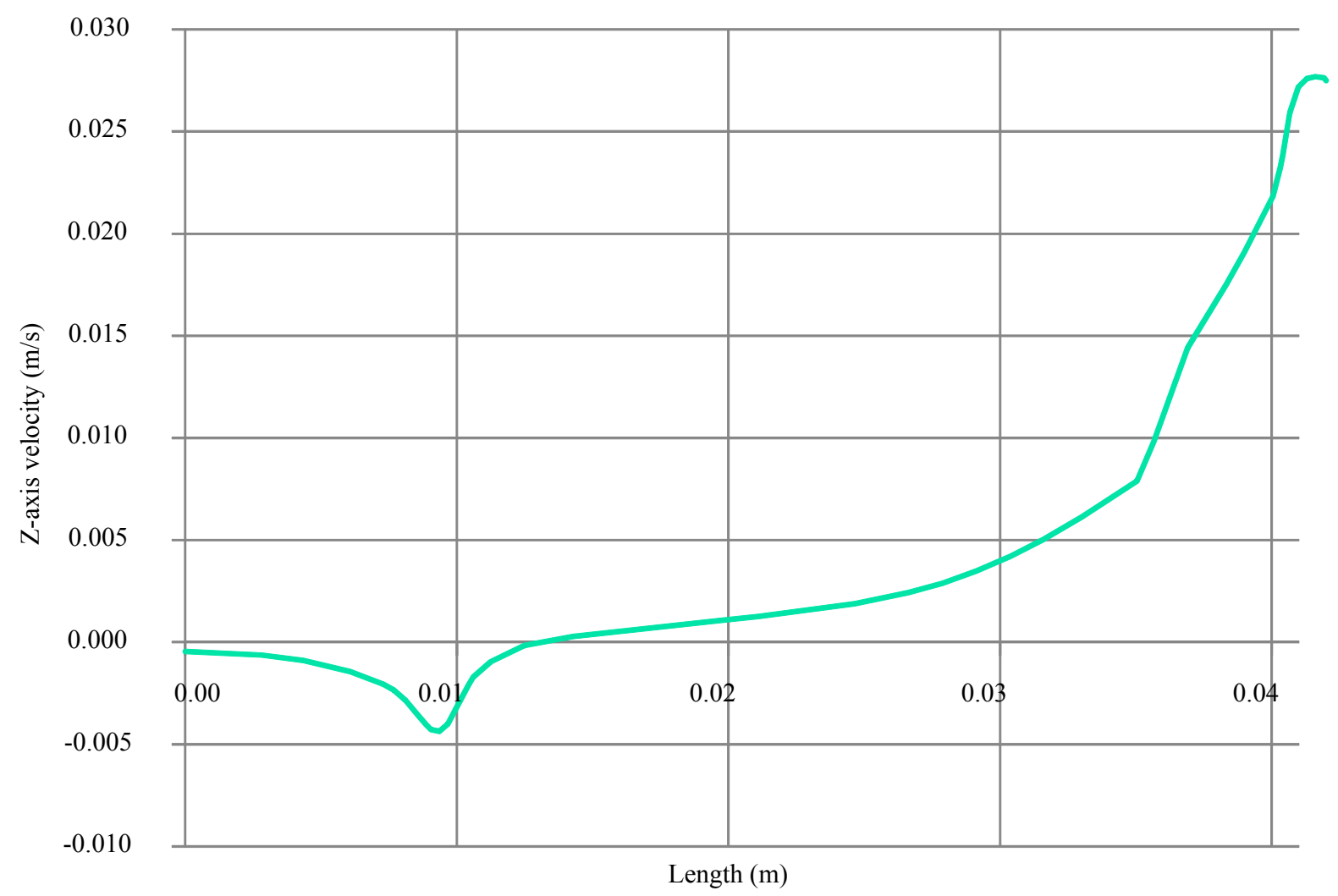

(b) Velocity profile at Z-axis

Fig. 8 Numerical results in the micro-pump in which the heat deformation of material is cooled. 
Fluid Flow Characteristics in Micro-Pump with the Aid of Peltier Devices and Thermal Expansion Material 101

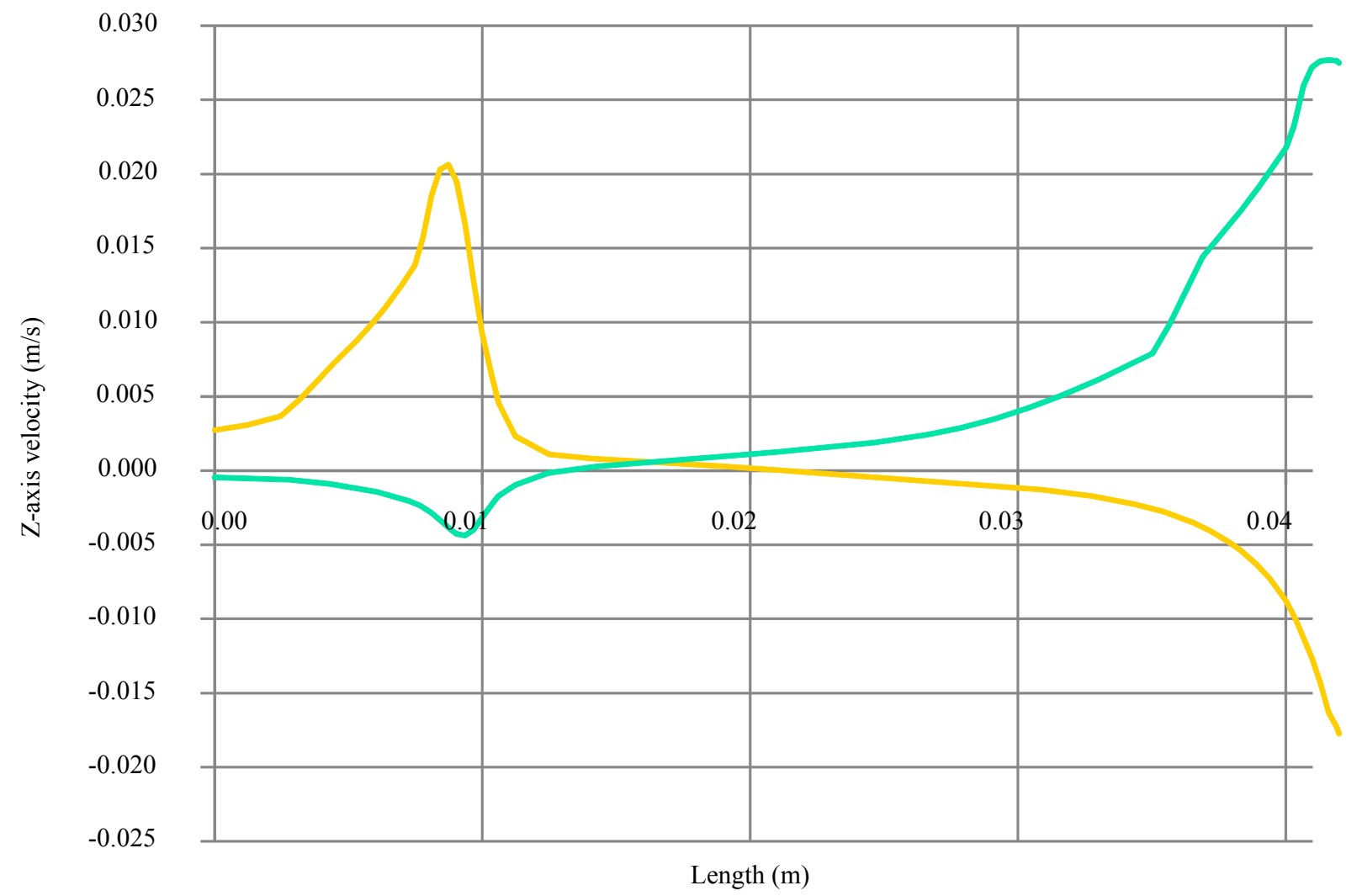

Fig. 9 Fluid flow characteristics along $\mathrm{X}$-axis in the micro-pump.

\section{Conclusions}

Experimental study has been performed on development and operation characteristics of the cylinder micropump, which are operated with heat deformation materials and Peltier devices. The following results are summarized:

(1) The PIV measurement with the aid of X-ray is possible through this study.

(2) The choice of particles becomes the important element for the flow visualization.

(3) Air bubble is better as the tracer particle in the case of the employment of X-ray.

\section{References}

[1] Terry, S. C. 1979. "A Gas Chromatographic Air Analyzer
Fabricated on a Silicon Wafer." IEEE Transaction on Electron Device 26 (12): 1880-6.

[2] Maruyama, S. 2006. Handbook of Heat and Fluid Engineering in Micro and Nano Scales. Kinokuniya: NTS Corporation, 378-97.

[3] van Lintel, H. T. G. 1979. "Piezoelectric Micropump Based on Micromachining of Silicon." Sensor. Actuat. 15 (2): 153-68.

[4] Stemme, E., and Stemme, G. 1993. "A Valveless Diffuser/Nozzle-Based Fluid Pump." Sensor. Actuat. A 39 (2): $159-67$.

[5] Tsai, J. H. 2002. "Thermal Bubble Actuated Nozzle-Diffuser Miro Pump.” Journal of Microelectromechanical Systems 11 (6): 665-71.

[6] Kline, S. J., and McClintock, F. A. 1953. "Describing Uncertainties in Single-Sample Experiments." Mechanical Engineering 75 (1): 3-8. 